\title{
Author Correction: Predicting the state of charge and health of batteries using data-driven machine learning
}

Man-Fai Ng, Jin Zhao, Qingyu Yan, Gareth J. Conduit and Zhi Wei Seh (D)

Correction to: Nature Machine Intelligence https://doi.org/10.1038/s42256-020-0156-7, published online 2 March 2020.

In the version of this Review originally published, the competing interests statement incorrectly read 'The authors declare no competing interests'; it should have read 'G.J.C. declares a potential financial conflict of interest as chief technology officer of Intellegens, UK'. This has now been corrected.

Published online: 12 June 2020

https://doi.org/10.1038/s42256-020-0191-4

(c) The Author(s), under exclusive licence to Springer Nature Limited 2020 